\title{
Analysis of the presence of bonding defects on the fracture behavior of a damaged plate repaired by composite patch
}

\author{
Kaddouri Nadia, Kouider Madani, Mohammed Amine Bellali \\ University of Djillali Liabes, LMPM Laboratory, Sidi Bel Abbes, Algeria \\ nadiia_171@hotmail.com,koumad10@yahoo.fr,bmaamine22@gmail.com
}

Xavier Feaugas

LASIE, UMR7356, Laboratory of Engineering Sciences for the Environment, La Rochelle University, La Rochelle, France. xavier.feaugas@univ-lr.fr

\begin{abstract}
The repair technique has presented its effectiveness in the reduction of the stresses at the level of stresses concentration areas. The search for a patch and suitable adhesive for a good transfer of load has pushed researchers to develop many ideas, which relate the form, the nature, the stacking sequence of the patch and the adhesive type to give a better combination of choice between the patch and the adhesive. Our work fits in this context; the objective is to analyze by the method of finite elements the behavior in the rupture of a damaged plate in the presence of defect of bonding. The analysis of J-Integral and stresses in the tow substrates adhesive and patch shows clearly that their values depends strongly on the position of the default essentially when it's located close to the free edge of the free edge of the adhesive or the crack.
\end{abstract}

KEYwORDS. J-Integral; Adhesive defect; Peel stress; Shear stress.

\section{OPEN ACCESS}

Citation: Kaddouri N., Madani, K., Bellali,M A., Feaugas, X,. Analysis of the presence of defects of bonding on the fracture behavior of a damaged plate repaired by composite patch, Frattura ed Integrità Strutturale, 49 (2019) 331-340.

Received: 26.02 .2019

Accepted: 02.06.2019

Published: 01.07.2019

Copyright: (C) 2019 This is an open access article under the terms of the CC-BY 4.0, which permits unrestricted use, distribution, and reproduction in any medium, provided the original author and source are credited.

\section{INTRODUCTION}

7 he use of the technique of bonding in the repair of damage in the aeronautical structures is experiencing a rapid expansion. This technique has shown its advantages compared to the classical technique such as welding or riveting and bolting. One of the most practical solutions for the repair of composite structures is to cover any part of the damaged area and paste patches in composite on the surface of the structure. It can also be noted that the bonding of external patches has been widely used in the repair of metallic structures in order to stop the propagation of a defect. Many studies have been redesigned for this type of repair [1].

Unfortunately, the repair of laminated composite structures by bonding external patches is much less concerned since it is limited because of its degradation towards environmental conditions $[2,3]$. 
The patch of composite material bonded on the outside face has been recognized as an effective method for repairing cracks, and consequently extend the service life of structures cracked. Considerable research has been done in recent years to develop the technology for bonding composite patches in aeronautical structures.

Several authors have searched to optimize the composite and the adhesive by the search for mechanical properties that are suitable to absorb as at the crack point is one of the approaches used in the performance analysis of composite material patches.

Experimental studies, numerical and even analytical, were conducted to study the behavior of cracks repaired by patch in composite. They showed that the crack point stress field, fatigue life and the stress intensity factor depend on several parameters, namely, the mechanical and geometrical properties, the number and thickness of the patch layers, the volume fraction and the orientation of the fibers, the shear modulus of the adhesive, its thickness and its temperature of elaboration and service, the size of much as possible the stresses of the damaged area. Other authors have searched to optimize the geometric shape of the patch in order to transfer more and more stress at the crack head or the damaged area [4].

Numerical analysis is an important tool for determining the stresses distribution in different substrates. Several authors have proposed many methods to model the repaired structure taking into account the mechanical parameters of the adhesive and the composite $[5,6]$.

The analysis of the stresses in the adhesive layer is important since this latter is the weakest link in the structure or its mechanical properties are the weakest and therefore rapid damage to the latter. However, little research has been devoted to studying the presence of bonding defects. $[7,8]$. The voids in the adhesive layer are produced during the treatment by the enclosed air or by gas products of the adhesive or adherents. The gas typically forms a bubble in the liquid adhesive and when the adhesive cross-links and solidifies, the bubble remains a void. In other hand, during the realization of a bonded assembly, many types of defects are likely to be created. The nature of the defects that can meet in the bonded assemblies varies according to the phenomena having caused these defects. The concentrations of porosities can lead to the creation of holes or cavities. When they are located in the close vicinity of the interface. The cavities create places of interfaces not related or geometric discontinuities. These may evolve and give birth to preferential sites of initiation of cracking or detachment leading to the rupture of the junction. Little research has been done in this area.

The objective of our study is to analyze by the finite element method the effect of the presence of a defect of square geometrical shape whose position is variable according to the surface of the adhesive on the value of the J-Integral and the different stress in the patch and the adhesive.

\section{GEOMETRICAL MODEL AND MECHANICAL PROPERTIES}

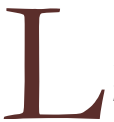

et consider a 2024-T3 Aluminum plate with the presence of a lateral crack repaired by a composite carbon / epoxy patch. This patch is bonded on the damaged area using an adhesive type ADEKIT A-140 as shown in Fig. 1.

The dimensions of the different substrates are shown in Tab. 1.

In order to analyze the effect of the presence of defect of bonding on the stresses transfer from the plate to the patch and therefore on the quality of the repair and the value of the J-Integral at the level of the crack tip, we have tried to model the presence of a defect in the adhesive layer. It was assumed a defect of Square shape of dimension $\left(1 * 1 \mathrm{~mm}^{2}\right)$ which its position is random. The most defect positions are chosen close to the crack, at the level of the crack and at the level of the adhesive free edges (Fig. 2).

\begin{tabular}{llll}
\hline & Length $(\mathrm{mm})$ & width $(\mathrm{mm})$ & Thickness $(\mathrm{mm})$ \\
Plate & $\mathrm{H}=250$ & $\mathrm{~W}=125$ & $\mathrm{e}_{\mathrm{p}}=2$ \\
Adhesif & $\mathrm{h}=80$ & $\mathrm{w}=80$ & $\mathrm{e}_{\mathrm{a}}=0.2$ \\
Patch & $\mathrm{h}=80$ & $\mathrm{w}=80$ & $\mathrm{e}_{\mathrm{r}}=2$ \\
\hline
\end{tabular}

Table 1: Dimensions of the different substrates.

The mechanical properties of the plate (2024-T3 Aluminum) and the adhesive (Adekit A-140) (Tab. 2) are drawn directly of traction tests performed in the laboratory LASIE (Laboratory of the Engineering sciences for the Environment) in France [9]. However, for the composite patch, the mechanical properties have been determined by calculation of 
homogenization between mechanical properties of matrix and fibers using the software CADEC designed for materials composites (see Tab. 2).

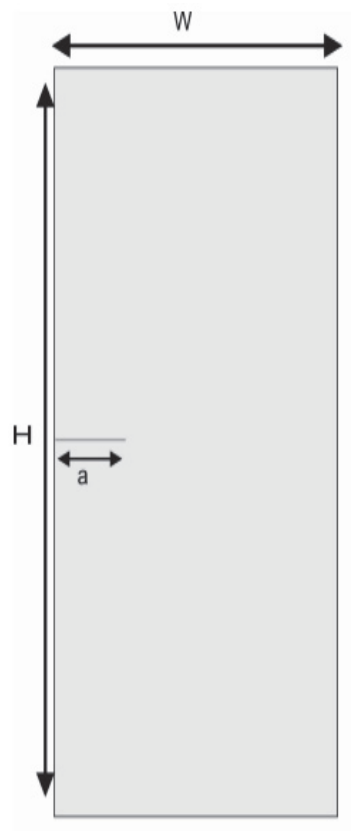

।
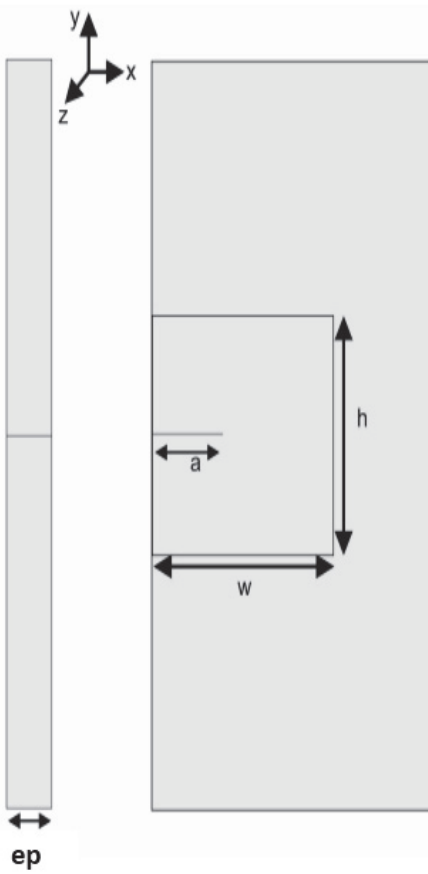

Figure 1: Geometric model.

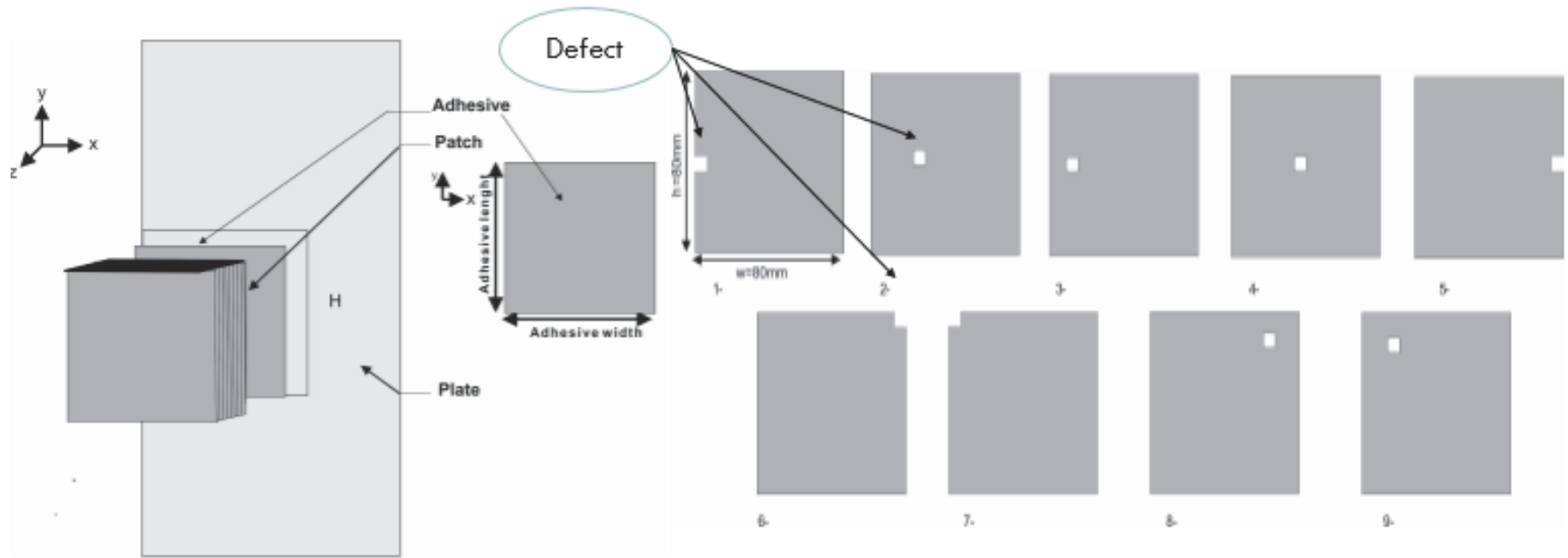

Figure 2: Some positions of adhesive defect.

\begin{tabular}{cccc}
\hline Materials & E: Young Modulus $(\mathrm{GPa})$ & $\mathrm{G}$ : Shear modulus $(\mathrm{GPa})$ & $\mathrm{v}$ : Poisson coefficient \\
Aluminum : 2024-T3 & 69 & 36,92 & 0,3 \\
Adhesive: Adekit A-140 & 2,690 & 0,99 & 0,3 \\
\hline
\end{tabular}

Table 2: Elastic properties of the Aluminum plate and adhesive [9].

The composite patch has been modeled as successive multilayer with different orientations. This modeling will allow us to introduce the mechanical properties real for each layer based on the directions of the fibers, which is more real. In this part, it has used the equations of homogenization to determine the mechanical characteristics of Composite (see Tab.3). 


\begin{tabular}{rlllllllll}
\hline Materials & $\mathrm{E}_{1}(\mathrm{MPa})$ & $\mathrm{E}_{2}(\mathrm{MPa})$ & $\mathrm{E}_{3}(\mathrm{MPa})$ & $\boldsymbol{v}_{\mathbf{1 2}}$ & $\boldsymbol{v}_{13}$ & $\boldsymbol{v}_{23}$ & $\mathrm{G}_{12}(\mathrm{MPa})$ & $\mathrm{G}_{13}(\mathrm{MPa})$ & $\mathrm{G}_{23}(\mathrm{MPa})$ \\
Carbon /Epoxy & 128600 & \multirow{2}{*}{9766} & 9766 & 0.346 & 0.346 & 0.346 & 5252 & 4364 & 4364 \\
\hline
\end{tabular}

Table 3: Mechanical properties of the composite patch Carbon/epoxy.

In order to see the effect of the fibers orientation in the composite patch on the global behavior of the repaired structure, six stacking sequences were selected as shown in the Tab. 4.

\begin{tabular}{cllllll}
\hline Laminates & $\mathrm{S} 1$ & $\mathrm{~S} 2$ & $\mathrm{~S} 3$ & $\mathrm{~S} 4$ & $\mathrm{~S} 5$ & \\
Stacking Sequence & {$\left[0_{8}\right]_{\mathrm{s}}$} & {$\left[\mathrm{O}_{2} / 15_{2} /-15_{2} / 90_{2}\right]_{\mathrm{s}}$} & {$\left[\mathrm{O}_{2} / 30_{2} /-30_{2} / 90_{2}\right]_{\mathrm{s}}$} & {$\left[0_{2} / 45_{2} /-45_{2} / 90_{2}\right]_{\mathrm{s}}$} & {$\left[0_{2} / 60_{2} /-60_{2} / 90_{2}\right]_{\mathrm{s}}$} & {$\left[0_{2} / 75_{2} /-75_{2} / 90_{2}\right]_{\mathrm{s}}$} \\
\hline
\end{tabular}

Table 4: Different stacking sequences used in the composite.

\section{MESH AND BOUNDARY CONDITION}

7 he boundary conditions for the repaired plate in traction are reproduced in the following manner:

- Embedding the lower face of the plate $\mathrm{u} 1=\mathrm{u} 2=\mathrm{u} 3=\mathrm{UR} 1=\mathrm{UR} 2=\mathrm{UR} 3=0$,

- Pressure at traction to the other face of the Amplitude $\mathrm{P}=50 \mathrm{MPa}$.

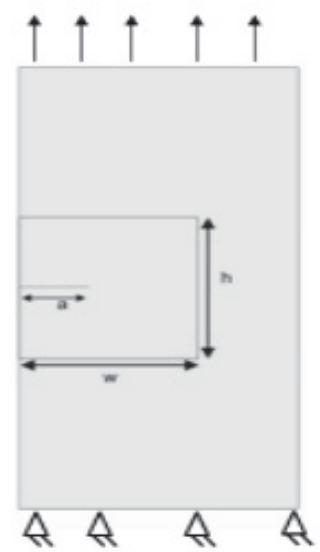

Figure 3: Model of the repaired structure under traction.

The numerical analysis has been carried out by the ABAQUS code using the finite elements methods. The structure been meshed by elements of hexagonal type linear C3D8R (Fig. 4). A refined mesh is carried out to the side of the crack and at the level of the patch and the adhesive; the adhesive has been considered as a third material so that one can introduce its mechanicals properties. The contact is considered as being perfect between the different layers (adhesive-patch, adhesiveplate and between the different layers of the composite). An elasto-plastic analysis is considered for the plate and the adhesive by introducing the plastic part of the two curves of the tensile test of the plate and the adhesive. For the composite, the mechanical properties has been varied the sequence of stacking (orientation of the fibers) and the Nature of the fibers, for this, one has used the CADEC code designed specially to determine the engineers constants of the composite.

\section{RESULTS AND ANALYSIS}

7 he Fig. 5 shows the variation of the J-Integral as a function of the length of the crack; we note clearly that the value of the J-Integral is low compared to the case of the plate unrepaired. It should be noted that the value of the J-Integral increases with the increase in the length of the crack. The slope of the curve becomes more acute and the value of the J-Integral increases. 


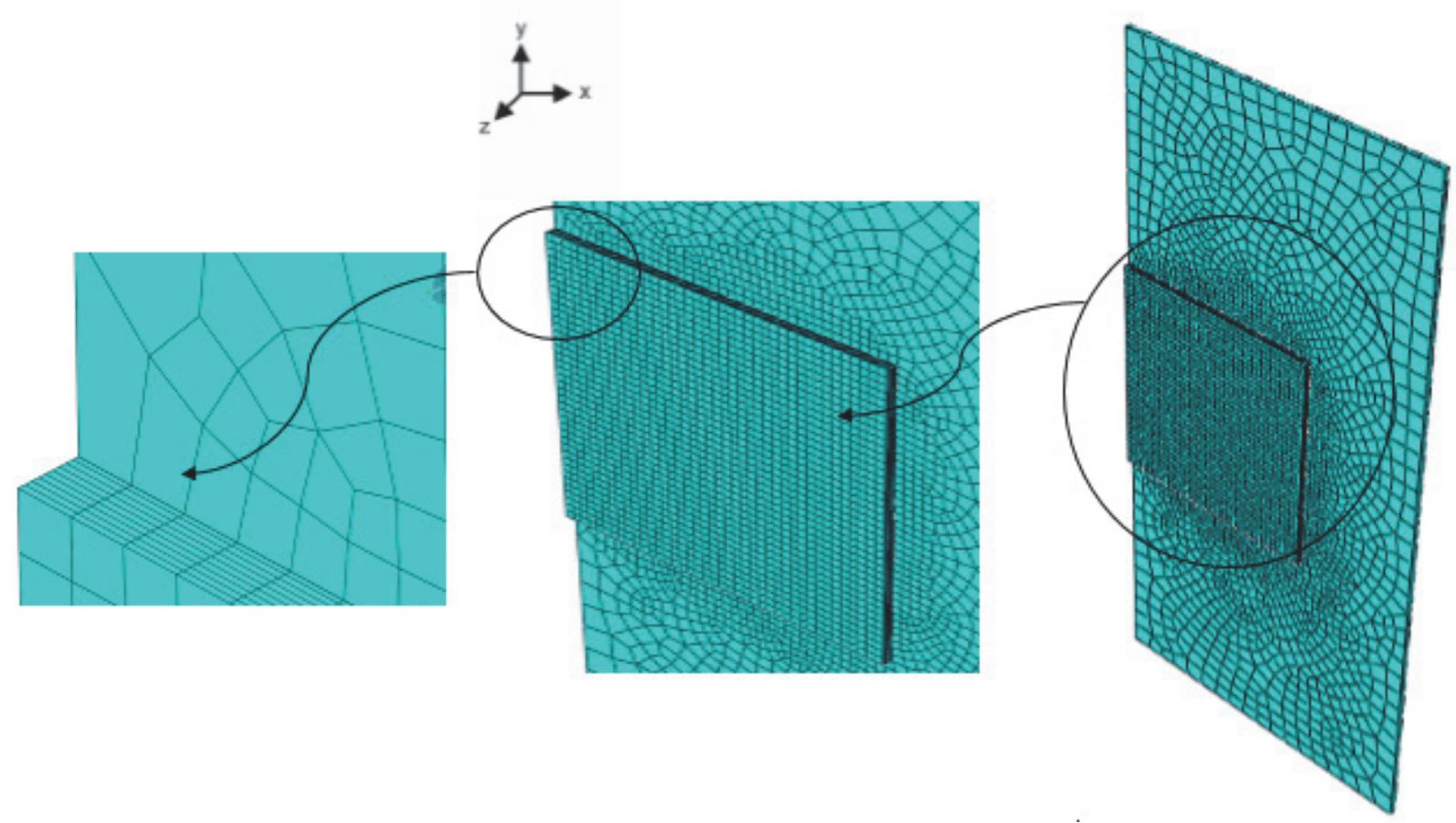

Figure 4: Mesh details of repaired plate.

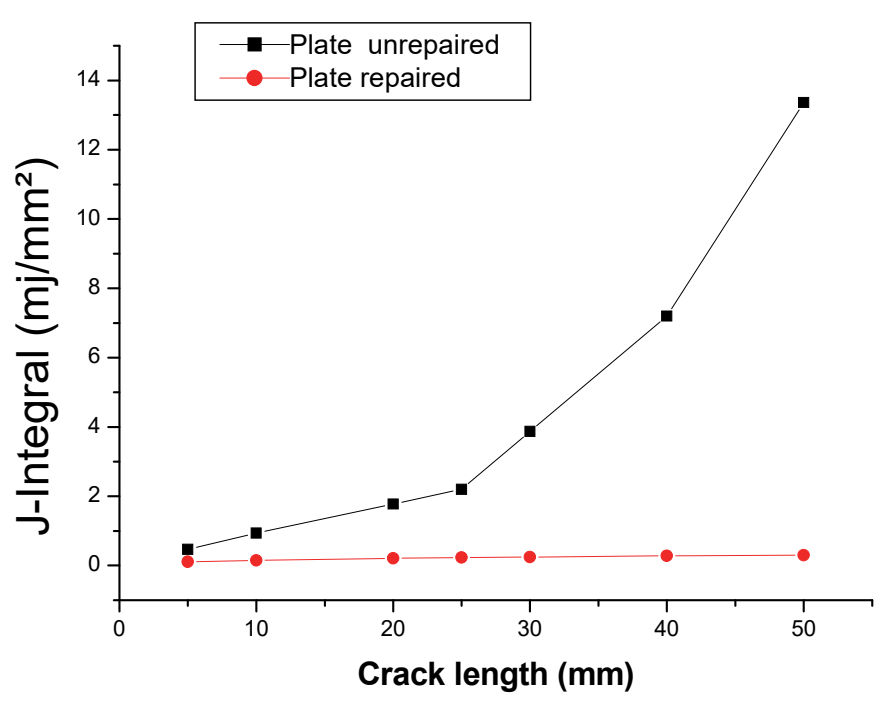

Figure 5: Variation of the J-Integral as a function of the length of the crack to the damaged plates and repaired by composite patch.

The choice of the laminate sequence for a patch is also important. For this we took 6 laminated with different fibers orientation ( see Tab. 4), the variation of the J-Integral is similar whatever the sequence of stacking, the value of the JIntegral is low for the patch that has the sequence $[0]_{8}$, this value increases if the sequence of the patch is $\left[0_{2} / 75_{2} /-\right.$ $\left.75_{2} / 90_{2}\right] \mathrm{s}$, for the laminate sequence $[0]_{8}$ the patch has the highest mechanical properties according to the loading direction and thus absorbed more stresses. However, if the fibers orientation angle tends toward $90^{\circ}$, the mechanical properties of the patch are declining and therefore less of load transfer from the damaged area.

If the length of the crack is too small than the width of the plate, the effect of the sequence of stacking disappears.

The J-Integral increases with the increase in the size of crack. The lowest values of the J-Integral are noted for the case of repair by patch for which the sequence of stacking is $\left[0_{8}\right] \mathrm{s}$, for this orientation the composite presents the mechanical properties high and therefore a better transfer of charge of the damaged plate through the adhesive. 


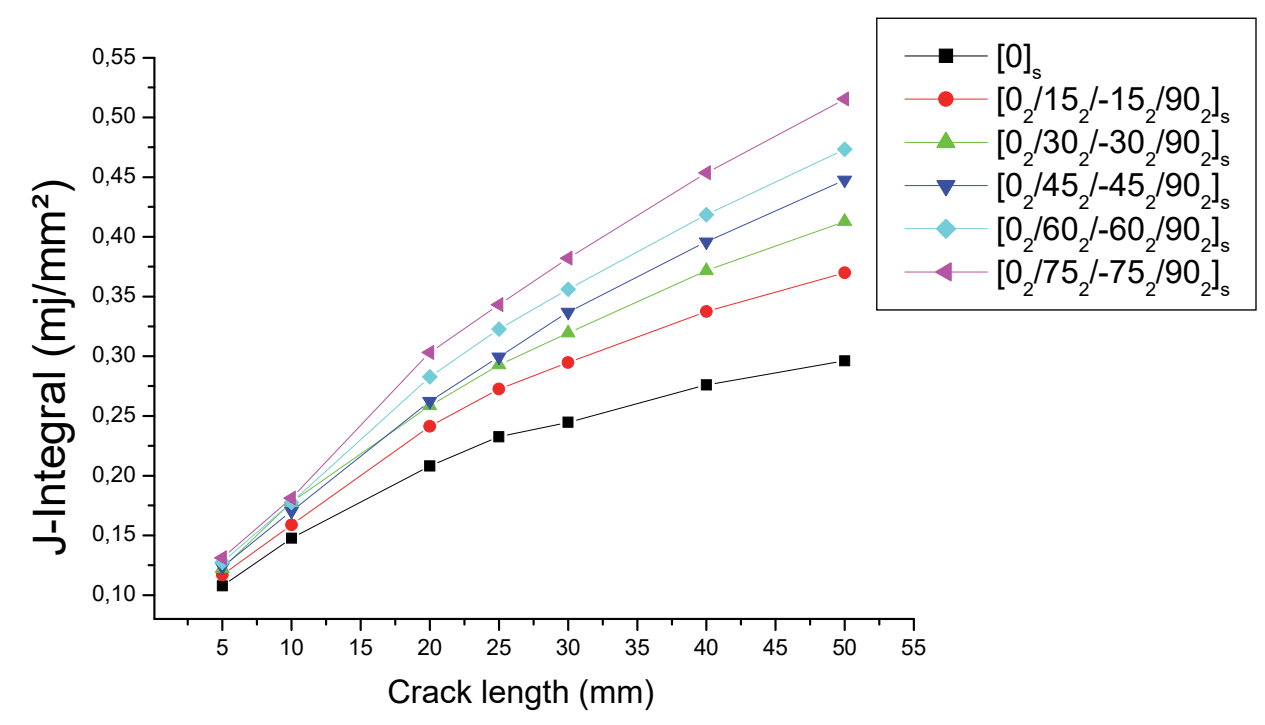

Figure 6: Variation J-Integral in function of the length of the crack for a different sequence of stacking.

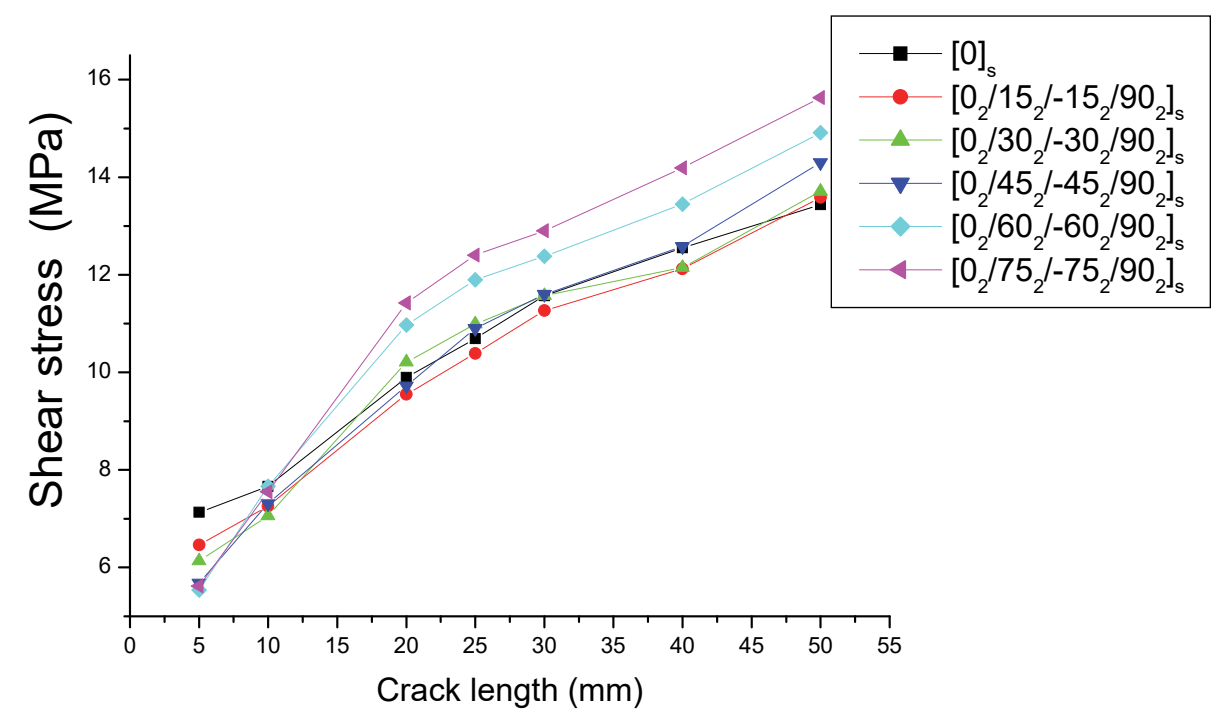

Figure 7: Variation of the adhesive Shear Stress $\tau_{23}$ in function of the the length of the crack for a different sequence of stacking.

The distribution of shear stresses is the same whatever the sequence of stacking in the composite. The more low stresses are for the patch with a sequence of stacking $\left[0_{8}\right] \mathrm{s}$ this is due to the fact that the majority of stresses in the Adhesive are absorbed by the patch.

For the other stacking sequences the transfer of stresses is minimal to the patch and therefore of high stresses in the adhesive.

By analyzing, the peel stress according to the thickness of the patch. If the length of the crack is minimum than the width of the patch, the peel stresses value is identical, a maximum is on the side in contact with the adhesive and a minimum on the other face which is in contact with the plate.

Only the sequence $\left[\mathrm{O}_{2}\right]_{8}$ presents a continuity in the peel stress distribution since the different layers present the same properties and the same orientation of fiber, however for the other stacking sequences the stress distribution is in the 
form of stairs since the mechanical properties of the different layers differ from a layer by report to the other depending on the direction of the fiber.
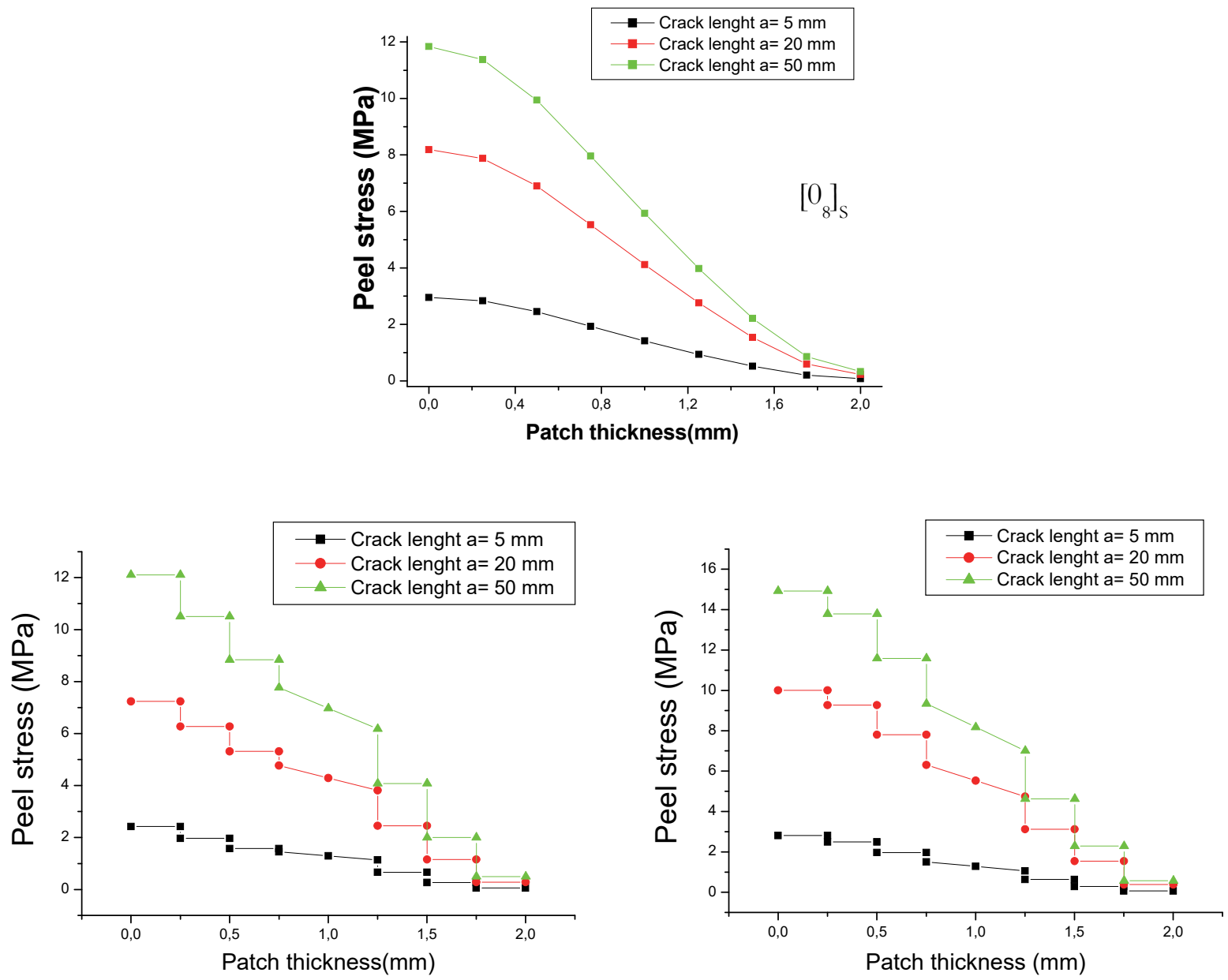

$\left[0{ }_{2} / 45_{2} /-45_{2} / 90\right]_{s}$

$\left[0{ }_{2} / 75_{2} /-75_{2} / 902\right]$

Figure 8: Variation of the Peel Stress $\sigma_{33}$ as a function of the thickness of the patch for different length of crack.

The value of the J-Integral increases considerably if the crack length increases.

If the defect is far from the crack and the free edge, the value of the J-Integral will be minimal, because, in these places, the presence of the defect will have no effect on the behavior of the adhesive. If the defect is at the level of the free edges of the adhesive, the value of the J-Integral will be affected since at these places the concentration of the stresses will be important. The defects that are at the core of the adhesive will have a consequence on the value of the J-Integral since they will be close to the crack.

These curves show the variation of the J-Integral as well as the constraints in the patch and the adhesive in function of the position of the defect. We can see clearly the positions, which induce maximum values of J-Integral, and stresses in the patch and the adhesive. The maximum values are for the case or the defect is located at the level of the free edge and a close to the crack. 

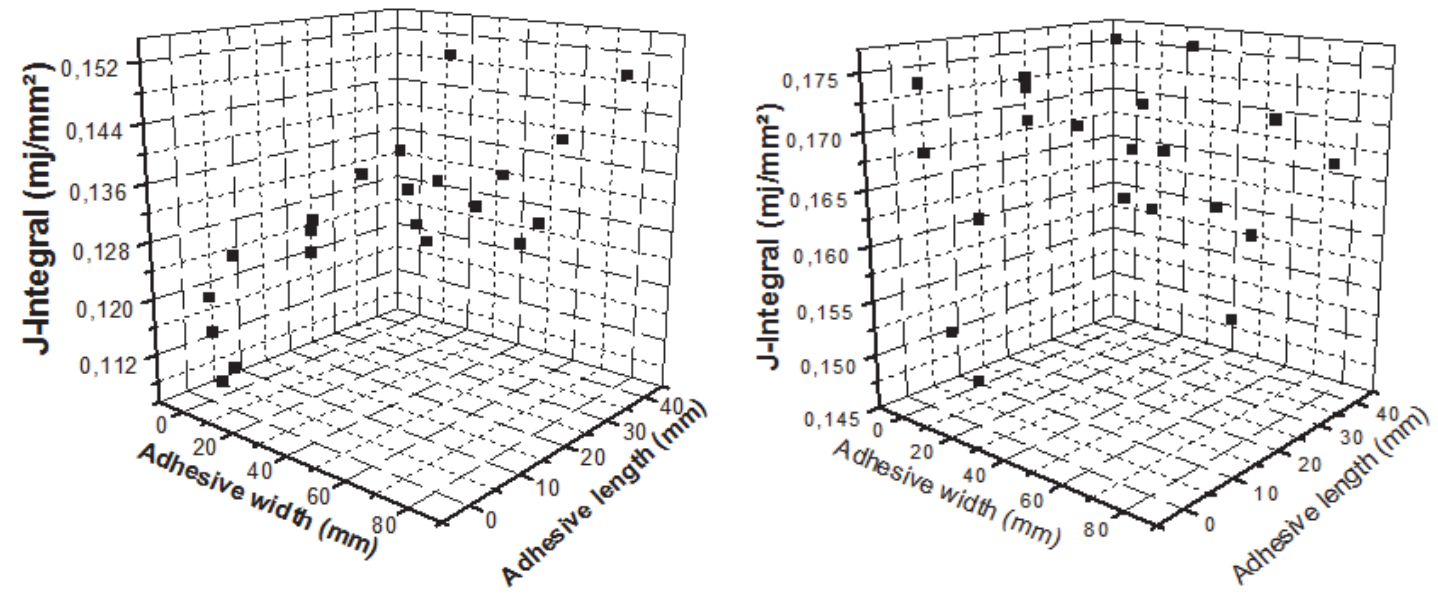

a)

b)

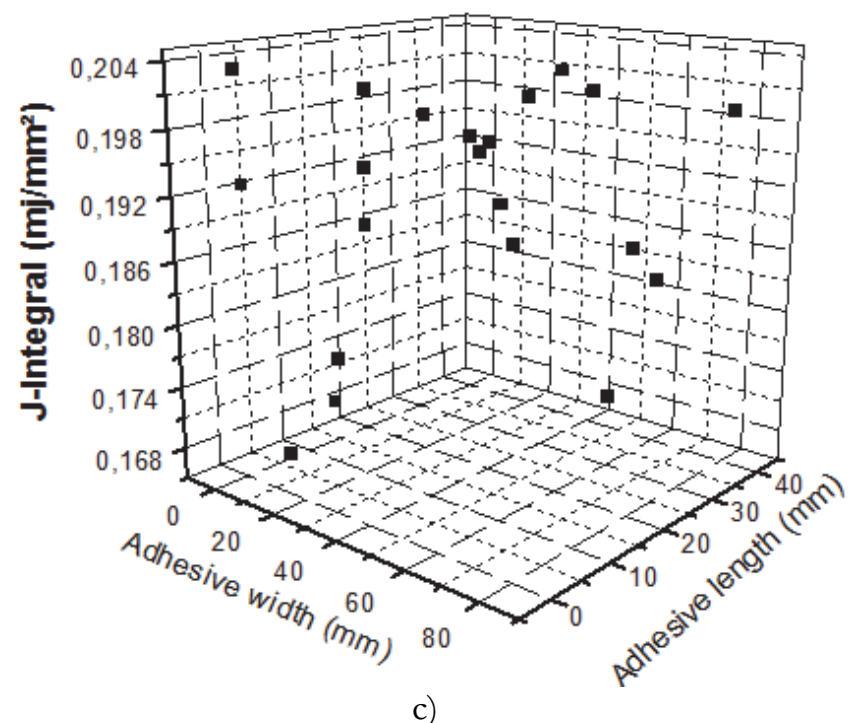

Figure 9: Variation of the J-Integral in function of the position of the default has a) Crack length $=10 \mathrm{~mm}, \mathrm{~b})$ Crack length $=20 \mathrm{~mm}$, c) Crack length $=30 \mathrm{~mm}$.

\section{Conclusion}

he analysis of the results allowed us to draw the following conclusions:

- The composite patch considerably reduces the J-Integral and the stresses in the different substrates.

- The stacking sequence plays a decisive role in reducing J-Integral and shear stresses in the adhesive and peeling in the patch.

- The presence of defect significantly affects the value of the J-Integral and the stresses in the two substrates.

- The presence of the defect of bonding, considerably affects the shear stress in the adhesive essentially if the defect is near the crack or at the free edges of the adhesive.

- The rate of charge transfer increases when stresses are high at the plate and the adhesive.

- The presence of the defect near a crack of considerable length considerably affects the stresses in the adhesive and J-Integral in the plate.

- If the length of the crack is minimal, the position of the defect has little influence on the value of J-Integral. 


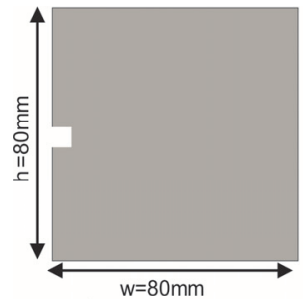

$1-$

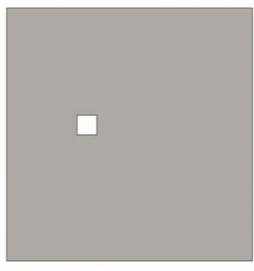

2-

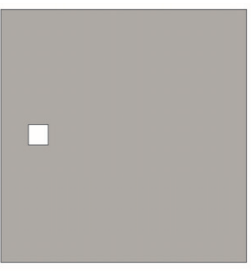

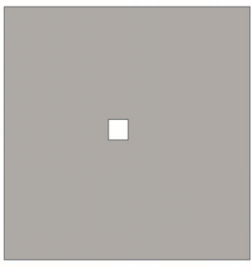

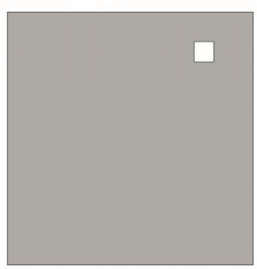

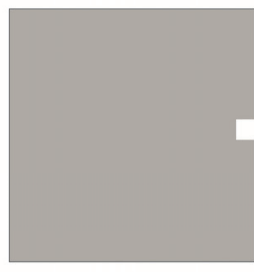

5

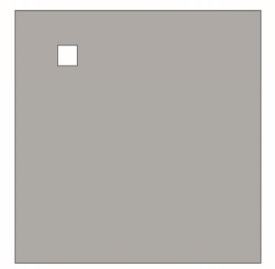

a)

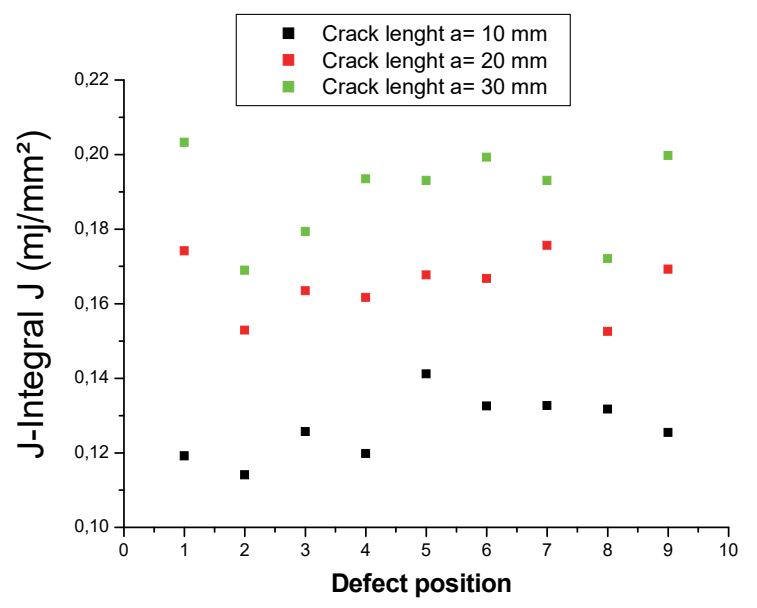

b)

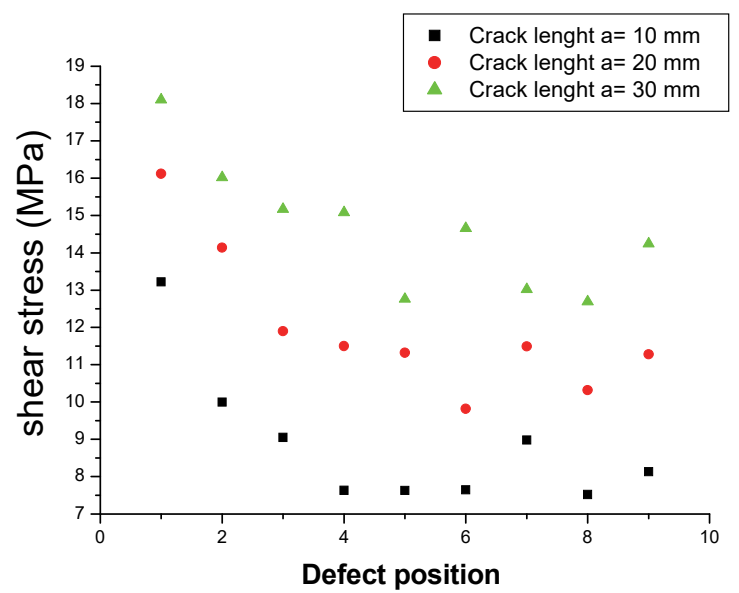

c)

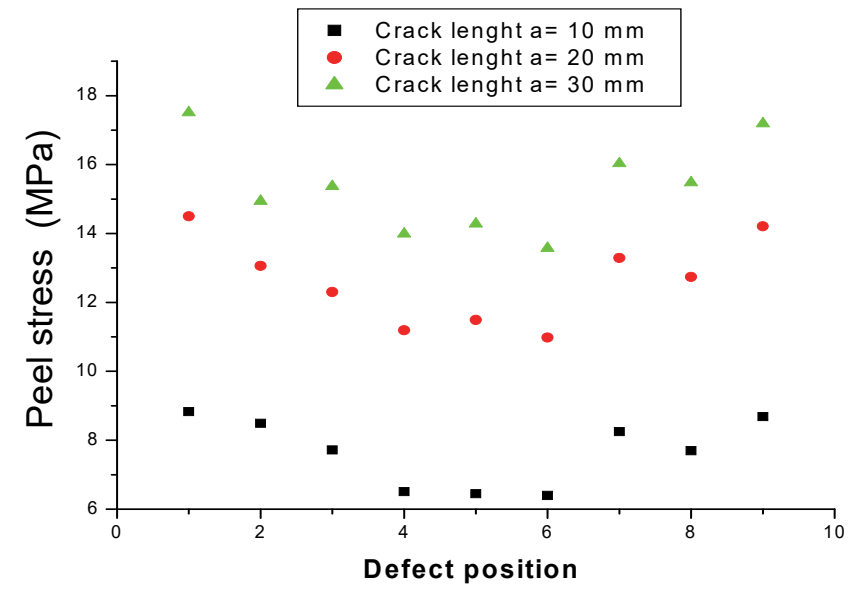

d)

Figure 10: a) Different position of default in the adhesive layer. b) Variation of the J-Integral in function of the position of the defect c) Variation of the Shear Stress as a function of the position of the defect. d) Variation of the Peel Stress according to the position of the defect. 


\section{REFERENCES}

[1] Madani, K., Touzain, S., Feaugas, X., Roy, A. a,d Cohendoz, S. (2009), Analyze of the notch effect on the distribution of the stresses in the adhesive layer between two bonded aluminum 2024-T3 plates. J Mater Technol; 97, pp. 315324.

[2] Rezgani, L. (2016), Influence of water ingress onto the crack propagation rate in an AA2024-T3 plate repaired by a carbon/epoxy patch. Aerospace Science and Technology.

[3] Rezgani, L. (2017), Hygrothermal ageing effect of ADEKIT A140 adhesive on the J-integral of a plate repaired by composite patch. Journal of Adhesion Science and Technology.

[4] Fekih, S. M. (2007), Optimization of geometric parameters and mechanical characteristics of patches in composites for the repair of cracked structures. University of SBA-UDL.

[5] Wu, Z.J., Romeijn, A., Wardenier, J. (1997), Stress expressions of single-lap adhesive joints of dissimilar adherends. Compos Struct 38, pp. 273.

[6] Kilic, B., Madenci, E., Ambur, D.R. (2006), Influence of adhesive spew in bonded single-lap joints. Eng FractMech 73, pp. 1472-1490.

[7] Elhannani, M. (2017), Statistical study of the influence of different bonding parameters on stress reduction.University of SBA-UDL.

[8] Benchiha, A. and Madani, K. (2015), Influence of the presence of defects on the stresses shear distribution in the adhesive layer for the single-lap bonded joint. Structural Engineering and Mechanics, 53(5).

[9] Madani, K., Touzain, S., Feaugas, X., Cohendouz, S., \& Ratwani, M. (2010). Experimental and numerical study of repair techniques for panels with geometrical discontinuities. Computational Materials Science, 48(1), pp. 83-93. DOI: 10.1016/j.commatsci.2009.12.005 . 